\title{
Gabapentin in Acute Postoperative Pain Management
}

\author{
Connie Y. Chang, Chaitanya K. Challa, Janki Shah, and Jean Daniel Eloy \\ Department of Anesthesia, Rutgers New Jersey Medical School, 185 South Orange Avenue, MSB E-538, Newark, NJ 07101-1709, USA
}

Correspondence should be addressed to Connie Y. Chang; cchang27@gmail.com

Received 26 December 2013; Revised 27 March 2014; Accepted 30 March 2014; Published 14 April 2014

Academic Editor: Alparslan Apan

Copyright (C) 2014 Connie Y. Chang et al. This is an open access article distributed under the Creative Commons Attribution License, which permits unrestricted use, distribution, and reproduction in any medium, provided the original work is properly cited.

\begin{abstract}
Gabapentin (1-aminomethyl-cyclohexaneacetic acid) is an amino acid that has the structure of the neurotransmitter $\gamma$-aminobutyric acid (GABA). It is a novel drug used for the treatment of postoperative pain with antihyperalgesic properties and a unique mechanism of action. Gabapentin and the related, more potent compound pregabalin have been shown to be beneficial in the treatment of neuropathic pain as well as postoperative pain following spinal surgery and hysterectomy. This study reviews five aspects of gabapentin: (1) chemical and structural characteristics; (2) pharmacokinetics and pharmacodynamics; (3) application in acute pain management; (4) adverse effects; and (5) drug safety. Overall, gabapentin has been reported to be a safe and efficacious drug for the treatment of postoperative pain.
\end{abstract}

\section{Introduction}

Primarily, three different classes of drugs are utilized for the treatment of postoperative pain (anti-inflammatories, local anesthetics, and opioids). Unfortunately long-term clinical use of these agents is limited by their side effects. Gabapentin is a novel drug used for the treatment of postoperative pain with antihyperalgesic properties and a unique mechanism of action, which differentiates it from other commonly used drugs. Various studies have shown that perioperative use of gabapentin reduces postoperative pain.

Gabapentin works by reducing lesion-induced hyperexcitability of posterior horn neurons, which is responsible for central sensitization [1]. The mechanism of the antihyperalgesic action may be a result of the postsynaptic binding of gabapentin to the alpha ${ }_{2}$-delta subunit of the dorsal horn neurons' voltage-dependent calcium channels, causing decreased calcium entry into nerve endings and thus decreased release of neurotransmitters. Other possible cellular mechanisms include the effects of gabapentin on NMDA receptors, sodium channels, monoaminergic pathways, and the opioid system [2-5].

Gabapentin was initially introduced in 1994 as an antiepileptic drug (AED), primarily for partial seizures. It is an anticonvulsant whose side effects are well tolerated and well absorbed after oral administration with the maximal plasma concentration seen after two to three hours [2, 6]. Some of the most commonly reported side effects of gabapentin include dizziness, somnolence, fatigue, ataxia, and peripheral edema $[4,7]$.

Gabapentin has also been found to be beneficial in treating neuropathic pain related to postherpetic neuralgia (PHN) $[8,9]$, postpoliomyelitis neuropathy $[10]$, and reflex sympathetic dystrophy [11]. Additionally, gabapentin has been shown to play a role in treating pain related to diabetic neuropathy [12] in placebo-controlled clinical trials.

This study reviews five aspects of gabapentin: (1) chemical and structural characteristics; (2) pharmacokinetics and pharmacodynamics; (3) application in acute pain management; (4) adverse effects; and (5) drug safety.

\section{Chemical and Structural Characteristics}

Gabapentin [1-(aminomethyl)cyclohexaneacetic acid] is a structural analog of the inhibitory neurotransmitter gammaaminobutyric acid (GABA) [13]. It is a white to off-white crystalline solid substance with the molecular formula, $\mathrm{C}_{9} \mathrm{H}_{17} \mathrm{NO}_{2}$, and a molecular weight of $171.237 \mathrm{~g} / \mathrm{mol}$. Freely soluble in water and in both basic and acidic aqueous solutions, gabapentin is highly charged at physiological $\mathrm{pH}$ and exists as a zwitterion with a $\mathrm{p} K_{\mathrm{a} 1}$ of 3.68 and $\mathrm{p} K_{\mathrm{a} 2}$ of 10.70 
[14]. Gabapentin is assayed in plasma and urine using gas chromatography [15], high performance liquid chromatography [16], and high performance liquid chromatography with UV-vis detection [17].

\section{Pharmacodynamics}

Though it is structurally similar to GABA, gabapentin does not bind to $\mathrm{GABA}_{A}$ or $\mathrm{GABA}_{B}$ receptors, does not block GABA uptake or metabolism, and has no direct GABAergic action [18]. The mechanism of action of gabapentin is different from that of several drugs that interact with GABA synapses such as valproate, barbiturates, and benzodiazepines; it is thought to exert its action by interaction with a receptor associated with the L-system amino acid carrier protein. In vitro studies with radiolabelled gabapentin have shown that gabapentin binds preferentially to neurons in the outer layer of the rat cortex and the hippocampus at sites distinct from other anticonvulsants [19]. Because the maximal anticonvulsant effect is seen 2 hours after an intravenous injection in rats, it is probable that gabapentin exerts its action at an intracellular site [20].

Gabapentin inhibits the tonic phase of nociception stimulated by formalin and carrageenan and in neuropathic pain models prevents mechanical and thermal allodynia and mechanical hyperalgesia [18]. Though the mechanism of action of gabapentin in the treatment of neuropathic pain is not clear, it does not influence the same pathways as opioids or tricyclic depressants. Current evidence indicates that gabapentin affects voltage-gated calcium channels in the CNS.

Gabapentin binds to the $\alpha_{2} \delta$ subunit of the voltagedependent calcium channel, regulating the action of the calcium channels and neurotransmitter release [15, 21, 22]. It is suggested that the antihyperalgesic action of gabapentin is due to its binding to this site on the voltage-gated calcium channel. Fink et al. demonstrated that gabapentin blocks neuronal calcium influx in a concentration-dependent manner by inhibiting P/Q-type calcium channels in the rat neocortex [23]. Decreased AMPA receptor activation and norepinephrine release in the brain are observed due to the reduced calcium influx, which decreases excitatory amino acid (glutamate) release [24]. These results support the hypothesis that the analgesic effects of gabapentin in neuropathic pain are mediated by the inhibition of voltagegated calcium channels.

Other effects of gabapentin that do not have major pharmacodynamic significance include slight reductions in the release of monoamine neurotransmitters (dopamine, norepinephrine, and serotonin) from mammalian brain tissue $[25,26]$ and a decrease in sodium-dependent action potentials, indicating sodium channel blockade, after prolonged exposure [27].

\section{Pharmacokinetics}

Administered orally, gabapentin is absorbed in part by diffusion and in part by the carrier-mediated, L-amino acid transport system [28]. As a result of the saturable transport mechanism, the bioavailability of gabapentin is inversely dependent on the dose [29]. It ranges from approximately $60 \%$ for a $300 \mathrm{mg}$ dose [30] to $40 \%$ for a $600 \mathrm{mg}$ dose [31] and $35 \%$ at steady state with doses of $1600 \mathrm{mg}$ three times daily [32]. The bioavailability of gabapentin is not affected by the presence of food and remains unchanged following multiple dose administration. Mean maximum plasma gabapentin concentrations $\left(C_{\max }\right)$ of $2.7 \pm 2.99 \mathrm{mg}$ per liter are reached in healthy volunteers approximately 3 hours after a single oral $300 \mathrm{mg}$ dose $[30,33]$. When the dose is tripled from $300 \mathrm{mg}$ to $900 \mathrm{mg}, C_{\max }$ increases less than threefold due to the dose dependent saturable absorption of gabapentin [32].

Gabapentin has a high volume of distribution of 0.6$0.8 \mathrm{~L} / \mathrm{kg}$ or $50-60 \mathrm{~L}$ in healthy volunteers $[30,32,33]$ and does not bind to human plasma proteins [34]. Unlike GABA, gabapentin readily penetrates the blood-brain barrier, yielding CSF concentrations equal to $20 \%$ of plasma concentrations and estimated between 0.09 and $0.14 \mu \mathrm{g}$ per $\mathrm{mL}[33,35]$. Brain tissue concentrations are $80 \%$ of corresponding plasma levels [36]. In rats, gabapentin concentrates in the pancreas and kidneys. Pancreatic and renal tissue concentrations are eight and four times higher than serum concentrations, respectively [37]. The drug does not accumulate in the pancreas in humans since it exists in a highly ionized state at physiological $\mathrm{pH}$ and concentrations in adipose tissue are low [37].

In contrast to many antiepileptic drugs, which are metabolized, gabapentin is not metabolized in humans and is eliminated solely by renal clearance. The drug is excreted unchanged in urine and undergoes first order kinetic elimination $[29,33]$. Plasma clearance of gabapentin is directly proportional to creatinine clearance; consequently, renal impairment reduces gabapentin excretion and increases plasma gabapentin concentrations in a linear fashion $[33,34,37,38]$. Patients with renal failure should receive their maintenance dose of gabapentin after each treatment as gabapentin is removed during hemodialysis [39]. Although a dose response pattern is apparent for plasma gabapentin concentrations and for clinical effect for doses ranging from 600 to $1800 \mathrm{mg}$ per day, it is not necessary to monitor plasma gabapentin concentrations and gabapentin dose should be modified based on clinical response [33]. The elimination half-life of gabapentin is between 5 and 9 hours, and as a result, three divided doses are usually required per day, but steady state is rapidly achieved [29].

Gabapentin is unique among anticonvulsant drugs as it lacks hepatic metabolism, exhibits low protein binding, and does not induce or inhibit hepatic microsomal enzymes or inhibit the metabolism of other antiepileptic drugs [29, 34]. No significant pharmacokinetic interactions have been reported between gabapentin and conventional antiepileptic drugs (valproic acid, phenobarbital, carbamazepine, or phenytoin) or oral contraceptives $[29,34]$. However, cimetidine, which decreases glomerular filtration rate, reduces the clearance of gabapentin by $12 \%$ [32]. In addition, the bioavailability of gabapentin is decreased by $20 \%$ by antacids when taken simultaneously or up to 2 hours after gabapentin administration [40]. 


\section{Application in Acute Pain Management}

5.1. Coronary Artery Bypass Graft. Ucak et al. evaluated the analgesic effects of perioperative gabapentin after coronary artery bypass graft (CABG) surgery with median sternotomy as well as internal mammary artery harvesting [41]. They used a gabapentin dose of $1.2 \mathrm{~g}$ per day treatment 1 hour before surgery and for 2 days after surgery and investigated its effect on postoperative acute pain. In this study, postoperative pain scores at 1,2, and 3 days as well as the consumption of tramadol which was given as rescue analgesic were significantly lower in the gabapentin group when compared to the placebo group [41].

5.2. Thoracic Surgery. Zakkar et al. performed a literature search and identified five papers with the best evidence regarding the use of gabapentin to reduce the incidence of pain experienced by patients after thoracic surgery [42-46]. They concluded that there is no evidence to support the role of a single preoperative oral dose of gabapentin in reducing pain scores or opioid consumption after thoracic surgery. Furthermore, more robust randomized control studies are needed to validate the efficacy of multiple dosing regimens but studies currently show that it may be beneficial in reducing acute pain [42].

5.3. Thyroid Surgery. Lee et al. explored the efficacy of using gabapentin $(600 \mathrm{mg}) 1$ hour before the administration of anesthesia for thyroid surgery [47]. He determined that the gabapentin group had a lower incidence of postoperative sore throat (POST) and a significantly lower visual analogue scale (VAS) score at 6 and 24 hours at rest after the completion of the surgery compared to the placebo group. However, there was no intergroup difference between the gabapentin group and the placebo group in terms of the incidence of POST or VAS score during the swallowing movement. From this study they were able to conclude that the gabapentin administered 1 hour prior to anesthesia decreased the intensity and incidence of POST at rest without a significant adverse event within the first 24 hours after thyroid surgery but not during the swallowing movement [47].

5.4. Neurological Surgery. Misra et al. performed a study to investigate patients undergoing craniotomies and the efficacy of gabapentin plus dexamethasone on postoperative nausea and vomiting (PONV) and pain after craniotomy [48]. Patients undergoing craniotomy received gabapentin $(600 \mathrm{mg})$ premedication orally 2 hours prior to induction of anesthesia as well as $4 \mathrm{mg}$ of intravenous dexamethasone on the morning of surgery and continued receiving it after every 8 hours. The 24 -hour incidence of nausea, emesis, or PONV and postoperative pain scores were evaluated. This study observed a significant difference between the group that received gabapentin and dexamethasone and the placebo group in the incidence of nausea and the requirements for antiemetics. However, there was no significant difference in either the postoperative pain scores or the opioid consumption between the gabapentin with dexamethasone cohort and the placebo cohort. Therefore, although there was no reduction in either the postoperative pain scores or opioid consumption, gabapentin plus dexamethasone significantly reduced the 24-hour incidence of nausea and PONV [48].

5.5. Lumbar Spinal Surgery. Yu et al. performed a systematic review and meta-analysis to determine the efficacy of gabapentin in the management of postoperative pain after lumbar spinal surgery. They showed that oral gabapentin was efficacious in the management of postoperative pain at every time point during the first day after surgery and therefore is efficacious in reducing postoperative pain and narcotic requirements after lumbar spinal surgery [49].

5.6. Hysterectomy. Ajori et al. performed a study investigating the preemptive use of gabapentin $(600 \mathrm{mg})$ prior to abdominal hysterectomy and its influence on nausea and vomiting, and meperidine consumption [50]. Pain was assessed on a visual analogue scale (VAS) at 1, 4, 6, 12, and 24 hours postoperatively. This study showed that gabapentin group had significantly lower VAS scores at every time interval compared to the placebo group and the total meperidine consumed in the gabapentin group was significantly less than in the placebo group. PONV and the consumption of antiemetic drugs were also significantly reduced in the gabapentin group. Therefore, preemptive use of $600 \mathrm{mg}$ gabapentin orally in patients undergoing abdominal hysterectomies significantly decreases postoperative pain and PONV and also reduces analgesic and antiemetic drug requirements [50].

5.7. Major Bowel Surgery. Siddiqui et al. performed a study investigating the effects of gabapentin $(600 \mathrm{mg})$ orally 1 hour prior to surgery in patients with inflammatory bowel disease (IBD) undergoing major bowel surgery. This group found that a single preoperative administration of $600 \mathrm{mg}$ gabapentin in patients undergoing major bowel surgery does not reduce postoperative pain scores, opioid consumption, or opioidrelated side effects [51].

5.8. Orthopedic Surgery. Panah Khahi et al. explored the efficacy of preemptive use of gabapentin on reduction of postoperative pain in the first 24 hours after internal fixation of the tibia under spinal anesthesia [52]. Patients were administered $300 \mathrm{mg}$ of gabapentin two hours before surgery and postoperative pain was evaluated using VAS two, 12 and 24 hours after surgery. The time from the completion of the surgery until the first bolus dose of morphine on demand and the total morphine required were also evaluated. This study showed that the pain score was significantly lower in the gabapentin group compared to the placebo group two hours after the completion of the surgery; however, the scores 12 and 24 hours after surgery were not significantly different between the two groups. Therefore, preemptive use of gabapentin $300 \mathrm{mg}$ orally significantly alleviated postoperative pain two hours after internal fixation of the tibia [52]. 


\section{Adverse Effects}

Gabapentin may have dose-limiting side effects, which could prevent some patients from achieving therapeutic plasma levels. Studies have reported no significant difference in the frequency of adverse events in patients receiving a higher dose as compared to those receiving a lower dose of gabapentin [53]. The most consistently cited adverse effects of gabapentin are somnolence and dizziness. While these side effects are considered to be minor, they may be worrisome in the elderly population, which is prone to injuries from falls and gait instability. Given this issue, clinicians may opt to discontinue gabapentin in clinical practice in this age group [54]. One possible explanation for these side effects is the multiple peaks and troughs in plasma concentration levels observed with treatment regimens consisting of multiple doses per day [54]. Since many clinical trials have shown that gabapentin demonstrates improvement in sleep, there may be an advantage for a formulation of gabapentin that peaks during the night, with lower levels during the day.

Clivatti et al. performed a study looking at twentysix randomized, placebo-controlled, clinical studies between 2005 and 2007 and evaluated the effects of gabapentin preoperatively (PRE Group) and pre- and postoperatively (PRE-POST Group) [55]. The study reported adverse effects which included postoperative nausea and vomiting, sedation, and dizziness. In the PRE Group, which consisted of seventeen studies, a reduction in the incidence of nausea and vomiting in patients treated with gabapentin was observed in two studies while an increase in nausea and vomiting was reported in one study. Additionally, a higher incidence of sedation was reported in one study while a higher incidence of dizziness was seen in another study. In the PRE-POST Group, which consisted of nine studies, one study reported an increase in the incidence of postoperative nausea and vomiting, another study reported an increase in sedation, and two studies reported an increase in the incidence of dizziness [55]. Likewise, a study by Fassoulaki et al. did not observe intolerable side effects with the use of gabapentin for the daily dose of $1600 \mathrm{mg} / \mathrm{d}$. Some sedation was exhibited by their patients, especially in the beginning of treatment, but that is desirable before and immediately after surgery.

Turan et al. investigated the effects of gabapentin on acute postoperative pain and on morphine consumption in patients undergoing spinal surgery where $1,200 \mathrm{mg}$ gabapentin was given 1 hour before surgery. The most common adverse effects of gabapentin observed during the study were dizziness and nausea but the number of incidences did not significantly differ from the placebo group and therefore no significant adverse effects were associated with a single oral dose of gabapentin [56]. However, in that same study, there was a significant decrease in vomiting and urinary retention in the gabapentin group versus the placebo group; therefore gabapentin decreased the side effects associated with morphine in patients undergoing spinal surgery [56].

Sen et al. showed that although the most common side effects after an elective hysterectomy were nausea and vomiting, there were no differences in the incidence of these side effects between the perioperative administration of a placebo, ketamine, and gabapentin [57]. Similarly, Gilron et al. assessed the efficacy and tolerability of gabapentin, nortriptyline, or a combination of both which showed that, both during dose titration and at the maximum tolerated dose, the most common adverse event was moderate or severe dry mouth, which occurred significantly less frequently in patients on gabapentin than on nortriptyline or combination treatment [58]. There were no other significant differences in adverse events [58].

Furthermore, a study by Compton et al. evaluated the efficacy of gabapentin, a key agent for neuropathic pain, to reverse opioid-induced hyperalgia in methadone maintenance for the treatment of addiction patients [59]. The two most commonly reported adverse events in this study were nausea and dizziness/lightheadedness which were both reported to be greater in the gabapentin group than in the placebo group [59].

A major complication that develops after the abrupt discontinuation of high-dose gabapentin used for the prevention of chronic postsurgical pain is withdrawal symptoms that clinically resemble alcohol or benzodiazepine withdrawal [60]. Signs and symptoms include irritability, agitation, anxiety, palpitation, and diaphoresis within 1-2 days. Secondary to gabapentin withdrawal, a patient with chronic back pain also developed generalized seizures and status epilepticus [61]. Tapering should always be performed especially in patients taking higher doses; however, withdrawal syndrome can still rarely be observed despite dose tapering [62].

\section{Pharmacovigilance}

Gabapentin has been reported to be a well-tolerated and safe drug $[12,33,63]$. Studies on safety issues have reported adverse effects including dizziness, somnolence, confusion, headache, nausea, ataxia, and weight gain $[53,64]$. However, these side effects were usually reported after long-term gabapentin use and usually diminish with time but may be bothersome in an acute setting to a postoperative patient. Studies which reported these side effects used the drug for 8 weeks to doses titrated up to $3600 \mathrm{mg}$ per day, unless severe adverse effects were developed.

Rowbotham et al. conducted a randomized controlled trial to determine the safety of gabapentin in reducing postherpetic neuralgia [63]. In this study, they used 229 subjects and performed a 4 -week titration period to maximum dose of $2600 \mathrm{mg}$ per day of gabapentin or matching placebo. The treatment was maintained for another 4 weeks at the maximum tolerated dose. They found that minor adverse events that were determined to be associated with the study medication were reported in 62 subjects receiving gabapentin and 32 subjects receiving placebo. The investigator reported no serious adverse events that were related to gabapentin. In this study, the most frequently reported adverse effects within the gabapentin group which were greater than the placebo group were somnolence (27.4\% versus 5.2\%), dizziness $(23.9 \%$ versus $5.2 \%)$, ataxia (7.1\% versus $0.0 \%)$, peripheral edema (9.7\% versus $3.4 \%)$, and infection (8.0\% versus $2.6 \%$ ) [63].

McLean et al. performed a study which evaluated and demonstrated the tolerability and safety of gabapentin when 
used as an adjunctive therapy in doses required to achieve the most effective seizure control in patients with epilepsy [53]. This study compared tolerability of gabapentin dosages less than or equal to $1,800 \mathrm{mg}$ versus those greater than $1,800 \mathrm{mg}$ per day. The safety analysis required patients to be followed up after receiving at least one dose of gabapentin and included 2,216 patients [53]. The safety of gabapentin therapy was evaluated by the following parameters: (1) adverse event reports, (2) physicians' assessment of safety and tolerability at study completion or termination, (3) neurologic examinations at baseline and study completion or termination, and (4) vital signs (sitting systolic and diastolic blood pressure, sitting heart rate) which were taken at baseline and study completion or termination. An adverse event was defined as a noxious or unintended event observed in or reported by a patient who was participating in the study and had received study medication and these adverse events were further classified relative to gabapentin therapy as definitely, probably, possibly, unlikely/remotely, or definitely not related. An associated adverse event was defined as an event that was considered by the investigator to be definitely, probably, or possibly related to treatment with gabapentin.

In this study, McLean et al. showed that $48.3 \%$ of patients reported the occurrence of at least one adverse event and $36.4 \%$ of patients reported at least one associated adverse event [53]. The most common adverse events for patients receiving less than or equal to $1,800 \mathrm{mg} /$ day were asthenia, headache, dizziness, and somnolence while the most common adverse events for patients receiving greater than $1,800 \mathrm{mg} /$ day were somnolence, dizziness, and weight gain. No adverse events were reported to occur at a significantly higher percentage of patients while receiving the higher dose compared to the lower dose of gabapentin. The rate of withdrawal from the study did not increase at higher doses suggesting that the incidence of adverse events does not increase at higher doses or longer use of gabapentin [53].

A serious adverse event was defined as any event that was fatal, life-threatening, and permanently disabling, required or prolonged inpatient hospitalization, or was a congenital anomaly, cancer, or overdose and was reported in only $3.3 \%$ of patients [53]. The most common serious adverse event was convulsion, reported by $0.9 \%$ of patients [53]. Eleven $(0.4 \%)$ patients died during the study although no deaths were considered to be associated with gabapentin treatment [53]. The safety and tolerability of gabapentin as determined by the physicians at the completion of the study or dropout were judged to be good or excellent for $78.5 \%$ of patients. Additionally, the treatment of gabapentin did not adversely affect vital signs in this study [53]. Overall, gabapentin was determined to be safe and doses greater than $1,800 \mathrm{mg} /$ day were well tolerated and not associated with more adverse events.

Backonja et al. investigated the effect of gabapentin monotherapy on pain associated with diabetic peripheral neuropathy by titrating from 900 to $3600 \mathrm{mg}$ per day or maximum tolerated dosage or placebo [12]. The safety of gabapentin was determined by using adverse event data (occurrence, intensity, and relationship to gabapentin) and the results of physical and neurological examination, including peripheral sensory examinations. During this study a total of seven gabapentin-treated patients (8\%) withdrew from the study due to adverse events which included dizziness and somnolence, abdominal pain, asthenia, body odor, headache, diarrhea, abnormal thinking, nausea, confusion, and hypesthesia. Out of the placebo group, only 5 patients (6\%) withdrew due to dyspepsia, constipation, flatulence infection, and somnolence. Most adverse events in patients treated with gabapentin were described as mild or moderate intensity. There were no significant changes in hemoglobin $A_{1 c}$ levels from baseline to the termination of treatment in either group. As such, glycemic control was maintained during the study. Neurological examination data revealed no significant differences in the rate of disease progression and the proportion of patients with a change from normal or decreased at baseline to absent at study termination was similar between groups (less than $14 \%$ in each case) for the 3 sensory modalities tested (temperature, light touch, and pin prick). Dizziness and somnolence were the two adverse events reported to occur more frequently in the gabapentin group. Overall, this study showed that gabapentin monotherapy produced rapid onset of clinical meaningful pain relief with relatively minor and potentially avoidable adverse effects; in turn, this further supports the safety of gabapentin as described in the prior studies [12].

\section{Conclusion}

Clinical studies have shown that gabapentin can reduce acute postoperative pain, decreasing the need for opioids. For example, a gabapentin dose of 1.2 grams per day 1 hour before surgery and for 2 days after CABG surgery showed that postoperative pain scores at 1,2, and 3 days as well as the consumption of tramadol given as a rescue analgesic were significantly lower in the gabapentin group when compared to the placebo group [41]. Additionally, preemptive use of gabapentin $300 \mathrm{mg}$ orally significantly alleviated postoperative pain two hours after internal fixation of the tibia. Similarly, preemptive use of $600 \mathrm{mg}$ gabapentin orally in patients undergoing abdominal hysterectomies significantly decreases postoperative pain and POVN and also reduces analgesic and antiemetic drug requirements [50]. While some studies suggest that gabapentin does not significantly reduce postoperative pain scores or opioid consumption, it has been shown to significantly reduce the 24-hour incidence of nausea and PONV. Overall, gabapentin has been reported to be a well-tolerated, safe, and efficacious drug.

\section{Conflict of Interests}

The authors declare that they have no conflict of interests.

\section{References}

[1] Y. P. Maneuf, M. I. Gonzalez, K. S. Sutton, F. Z. Chung, R. D. Pinnock, and K. Lee, "Cellular and molecular action of the putative GABA-mimetic, gabapentin," Cellular and Molecular Life Sciences, vol. 60, no. 4, pp. 742-750, 2003. 
[2] B. Gidal and R. Billington, "New and emerging treatment options for neuropathic pain," The American Journal of Managed Care, vol. 12, pp. S269-S278, 2006.

[3] C. P. Taylor, N. S. Gee, T. Z. Su et al., "A summary of mechanistic hypotheses of gabapentin pharmacology," Epilepsy Research, vol. 29, no. 3, pp. 233-249, 1998.

[4] J. B. Dahl, O. Mathiesen, and S. Møiniche, "'Protective premedication': an option with gabapentin and related drugs? A review of gabapentin and pregabalin in the treatment of post-operative pain," Acta Anaesthesiologica Scandinavica, vol. 48, no. 9, pp. 1130-1136, 2004.

[5] G. J. Sills, "Not another gabapentin mechanism!" Epilepsy Currents, vol. 5, no. 2, pp. 75-77, 2005.

[6] R. D. C. Elwes and C. D. Binnie, "Clinical pharmacokinetics of newer antiepileptic drugs. Lamotrigine, vigabatrin, gabapentin and oxcarbazepine," Clinical Pharmacokinetics, vol. 30, no. 6, pp. 403-415, 1996.

[7] J. D. Markman and R. H. Dworkin, "Ion channel targets and treatment efficacy in neuropathic pain," Journal of Pain, vol. 7, pp. S38-S47, 2006.

[8] A. Z. Segal and G. Rordorf, "Gabapentin as a novel treatment for postherpetic neuralgia," Neurology, vol. 46, no. 4, pp. 1175-1176, 1996.

[9] H. Rosner, L. Rubin, and A. Kestenbaum, "Gabapentin adjunctive therapy in neuropathic pain states," Clinical Journal of Pain, vol. 12, no. 1, pp. 56-58, 1996.

[10] J. J. Zapp, "Postpoliomyelitis pain treated with gabapentin," The American Family Physician, vol. 53, pp. 2442-2445, 1996.

[11] G. A. Mellick and L. B. Mellick, "Reflex sympathetic dystrophy treated with gabapentin," Archives of Physical Medicine and Rehabilitation, vol. 78, no. 1, pp. 98-105, 1997.

[12] M. Backonja, A. Beydoun, K. R. Edwards et al., "Gabapentin for the symptomatic treatment of painful neuropathy in patients with diabetes mellitus. A randomized controlled trial," Journal of the American Medical Association, vol. 280, no. 21, pp. 18311836, 1998.

[13] K. Y. Ho, T. J. Gan, and A. S. Habib, "Gabapentin and postoperative pain-a systematic review of randomized controlled trials," Pain, vol. 126, no. 1-3, pp. 91-101, 2006.

[14] G. D. Bartoszyk, N. Meyerson, W. Reimann, G. Satzinger, and A. von Hodenberg, "Gabapentin," in New Anticonvulsant Drugs, B. S. Meldrum and B. J. Porter, Eds., pp. 147-163, John Libbey, London, UK, 1986.

[15] W. D. Hooper, M. C. Kavanagh, and R. G. Dickinson, "Determination of gabapentin in plasma and urine by capillary column gas chromatography," Journal of Chromatography, vol. 529, no. 1, pp. 167-174, 1990.

[16] H. Hengy and E. U. Kolle, "Determination of gabapentin in plasma and urine by high-performance liquid chromatography and pre-column labelling for ultraviolet detection," Journal of Chromatography, vol. 341, no. 2, pp. 473-478, 1985.

[17] O. Sagirli, S. M. Çetin, and A. Önal, "Determination of gabapentin in human plasma and urine by high-performance liquid chromatography with UV-vis detection," Journal of Pharmaceutical and Biomedical Analysis, vol. 42, no. 5, pp. 618-624, 2006.

[18] M. A. Rose and P. C. A. Kam, "Gabapentin: pharmacology and its use in pain management," Anaesthesia, vol. 57, no. 5, pp. 451$462,2002$.
[19] D. R. Hill, N. Suman-Chauhan, and G. N. Woodruff, "Localization of $[3 \mathrm{H}]$ gabapentin to a novel site in rat brain: autoradiographic studies," European Journal of Pharmacology, vol. 244, no. 3, pp. 303-309, 1993.

[20] D. F. Welty, G. P. Schielke, M. G. Vartanian, and C. P. Taylor, "Gabapentin anticonvulsant action in rats: Disequilibrium with peak drug concentrations in plasma and brain microdialysate," Epilepsy Research, vol. 16, no. 3, pp. 175-181, 1993.

[21] B. J. Partridge, S. R. Chaplan, E. Sakamoto, and T. L. Yaksh, "Characterization of the effects of gabapentin and 3-isobutyl- $\gamma$ aminobutyric acid on substance P-induced thermal hyperalgesia," Anesthesiology, vol. 88, no. 1, pp. 196-205, 1998.

[22] A. B. Malmberg and T. L. Yaksh, "Voltage-sensitive calcium channels in spinal nociceptive processing: blockade of $\mathrm{N}$ - and Ptype channels inhibits formalin-induced nociception," Journal of Neuroscience, vol. 14, no. 8, pp. 4882-4890, 1994.

[23] K. Fink, W. Meder, D. J. Dooley, and M. Göthert, "Inhibition of neuronal $\mathrm{Ca}^{2+}$ influx by gabapentin and subsequent reduction of neurotransmitter release from rat neocortical slices," British Journal of Pharmacology, vol. 130, no. 4, pp. 900-906, 2000.

[24] D. J. Dooley, C. A. Mieske, and S. A. Borosky, "Inhibition of $\mathrm{K}^{+}$evoked glutamate release from rat neocortical and hippocampal slices by gabapentin," Neuroscience Letters, vol. 280, no. 2, pp. 107-110, 2000.

[25] W. Reimann, "Inhibition by GABA, baclofen and gabapentin of dopamine release from rabbit caudate nucleus: are there common or different sites of action?" European Journal of Pharmacology, vol. 94, no. 3-4, pp. 341-344, 1983.

[26] E. Schlicker, W. Reimann, and M. Gothert, "Gabapentin decreases monoamine release without affecting acetylcholine release in the brain," Arzneimittel-Forschung/Drug Research, vol. 35, no. 9, pp. 1347-1349, 1985.

[27] A. W. Wamil and M. J. McLean, "Limitation by gabapentin of high frequency action potential firing by mouse central neurons in cell culture," Epilepsy Research, vol. 17, no. 1, pp. 1-11, 1994.

[28] B. H. Stewart, A. R. Kugler, P. R. Thompson, and H. N. Bockbrader, "A saturable transport mechanism in the intestinal absorption of gabapentin is the underlying cause of the lack of proportionality between increasing dose and drug levels in plasma," Pharmaceutical Research, vol. 10, no. 2, pp. 276-281, 1993.

[29] M. J. McLean, “Clinical pharmacokinetics of gabapentin,” Neurology, vol. 44, pp. S17-S22, 1994.

[30] K. O. Vollmer, H. Anhut, P. Thomann, F. Wagner, and D. Jahncken, "Pharmacokinetic model and absolute bioavailability of the new anticonvulsant gabapentin," Advances in Epileptology, vol. 17, pp. 209-211, 1989.

[31] D. Turck VK, H. Bockbrader, and A. Sedman, "Dose-linearity of the new anticonvulsant gabapentin after multiple oral doses," European Journal of Clinical Pharmacology, vol. 36, supplement A310, pp. 8-11, 1989.

[32] T. O. Baldwin, M. M. Ziegler, A. F. Chaffotte, and M. E. Goldberg, "Contribution of folding steps involving the individual subunits of bacterial luciferase to the assembly of the active heterodimeric enzyme," Journal of Biological Chemistry, vol. 268, no. 15, pp. 10766-10772, 1993.

[33] K. L. Goa and E. M. Sorkin, "Gabapentin: a review of its pharmacological properties and clinical potential in epilepsy," Drugs, vol. 46, no. 3, pp. 409-427, 1993.

[34] A. Beydoun, B. M. Uthman, and J. C. Sackellares, "Gabapentin: Pharmacokinetics, efficacy, and safety," Clinical Neuropharmacology, vol. 18, no. 6, pp. 469-481, 1995. 
[35] E. Ben-Menachem, L. I. Persson, and T. Hedner, "Selected CSF biochemistry and gabapentin concentrations in the CSF and plasma in patients with partial seizures after a single oral dose of gabapentin," Epilepsy Research, vol. 11, no. 1, pp. 45-49, 1992.

[36] L. M. Ojemann, P. N. Friel, and G. A. Ojemann, "Gabapentin concentrations in human brain," Epilepsia, vol. 29, p. 694, 1988.

[37] K.-O. Vollmer, A. Von Hodenberg, and E. U. Kolle, "Pharmacokinetics and metabolism of gabapentin in rat, dog and man," Arzneimittel-Forschung, vol. 36, no. 5, pp. 830-839, 1986.

[38] R. A. Blum, T. J. Comstock, D. A. Sica et al., "Pharmacokinetics of gabapentin in subjects with various degrees of renal function," Clinical Pharmacology and Therapeutics, vol. 56, no. 2, pp. 154-159, 1994.

[39] M. O. Wong, M. A. Eldon, W. F. Keane et al., "Disposition of gabapentin in anuric subjects on hemodialysis," Journal of Clinical Pharmacology, vol. 35, no. 6, pp. 622-626, 1995.

[40] J. A. Busch RL, L. Radulovic, H. N. Bockbrader, B. A. Underwood, A. J. Sedman, and T. Chang, "Effect of Maalox TC on single- dose pharmacokinetics of gabapentin capsules in healthy subjects," Pharmaceutical Research, vol. 9, supplement, p. S315, 1992.

[41] A. Ucak, B. Onan, H. Sen, I. Selcuk, A. Turan, and A. T. Yilmaz, "The effects of gabapentin on acute and chronic postoperative pain after coronary artery bypass graft surgery," Journal of Cardiothoracic and Vascular Anesthesia, vol. 25, no. 5, pp. 824829, 2011.

[42] M. Zakkar, S. Frazer, and I. Hunt, "Is there a role for Gabapentin in preventing or treating pain following thoracic surgery?" Interactive Cardiovascular and Thoracic Surgery, vol. 17, pp. 716719, 2013.

[43] M. P. Huot, P. Chouinard, F. Girard, M. Ruel, E. R. Lafontaine, and P. Ferraro, "Gabapentin does not reduce post-thoracotomy shoulder pain: a randomized, double-blind placebo-controlled study," Canadian Journal of Anesthesia, vol. 55, no. 6, pp. 337343, 2008.

[44] M. A. O. Kinney, C. B. Mantilla, P. E. Carns et al., "Preoperative gabapentin for acute post-thoracotomy analgesia: a randomized, double-blinded, active placebo-controlled study," Pain Practice, vol. 12, no. 3, pp. 175-183, 2012.

[45] A. D. Sihoe, T. W. Lee, I. Y. P. Wan, K. H. Thung, and A. P. Yim, "The use of gabapentin for post-operative and post-traumatic pain in thoracic surgery patients," European Journal of Cardiothoracic Surgery, vol. 29, no. 5, pp. 795-799, 2006.

[46] O. Solak, M. Metin, H. Esme et al., "Effectiveness of gabapentin in the treatment of chronic post-thoracotomy pain," European Journal of Cardio-thoracic Surgery, vol. 32, no. 1, pp. 9-12, 2007.

[47] J. H. Lee, H. K. Lee, N. H. Chun, Y. So, and C. Y. Lim, "The prophylactic effects of gabapentin on postoperative sore throat after thyroid surgery," Korean Journal of Anesthesiology, vol. 64, pp. 138-142, 2013.

[48] S. Misra, G. Parthasarathi, and G. C. Vilanilam, "The effect of gabapentin premedication on postoperative nausea, vomiting, and pain in patients on preoperative dexamethasone undergoing craniotomy for intracranial tumors," Journal of Neurosurgical Anesthesiology, vol. 25, pp. 386-391, 2013.

[49] L. Yu, B. Ran, M. Li, and Z. Shi, "Gabapentin and pregabalin in the management of postoperative pain after lumbar spinal surgery: a systematic review and meta-analysis," Spine, vol. 38, no. 22, pp. 1947-1952, 2013.

[50] L. Ajori, L. Nazari, M. M. Mazloomfard, and Z. Amiri, "Effects of gabapentin on postoperative pain, nausea and vomiting after abdominal hysterectomy: a double blind randomized clinical trial," Archives of Gynecology and Obstetrics, vol. 285, pp. 677682, 2012.

[51] N. T. Siddiqui, H. Fischer, L. Guerina, and Z. Friedman, "Effect of a preoperative gabapentin on postoperative analgesia in patients with inflammatory bowel disease following major bowel surgery: a randomized, placebo-controlled trial," Pain Practice, vol. 14, no. 2, pp. 132-139, 2013.

[52] M. Panah Khahi, A. A. Yaghooti, S. H. Marashi, and A. Nadjafi, "Effect of pre-emptive gabapentin on postoperative pain following lower extremity orthopaedic surgery under spinal anaesthesia," Singapore Medical Journal, vol. 52, no. 12, pp. 879-882, 2011.

[53] M. J. McLean, M. J. Morrell, L. J. Willmore et al., "Safety and tolerability of gabapentin as adjunctive therapy in a large, multicenter study," Epilepsia, vol. 40, no. 7, pp. 965-972, 1999.

[54] B. Beal, T. Moeller-Bertram, J. M. Schilling, and M. S. Wallace, "Gabapentin for once-daily treatment of post-herpetic neuralgia: a review," Clinical Interventions in Aging, vol. 7, pp. 249-255, 2012.

[55] J. Clivatti, R. K. Sakata, and A. M. Issy, "Review of the use of gabapentin in the control of postoperative pain," Revista Brasileira de Anestesiologia, vol. 59, no. 1, pp. 87-98, 2009.

[56] A. Turan, B. Karamanlioǧlu, D. Memiş et al., "Analgesic Effects of Gabapentin after Spinal Surgery," Anesthesiology, vol. 100, no. 4, pp. 935-938, 2004.

[57] H. Sen, A. Sizlan, O. Yanarates et al., "A comparison of gabapentin and ketamine in acute and chronic pain after hysterectomy," Anesthesia and Analgesia, vol. 109, no. 5, pp. 1645-1650, 2009.

[58] I. Gilron, J. M. Bailey, D. Tu, R. R. Holden, A. C. Jackson, and R. L. Houlden, "Nortriptyline and gabapentin, alone and in combination for neuropathic pain: a double-blind, randomised controlled crossover trial," The Lancet, vol. 374, no. 9697, pp. 1252-1261, 2009.

[59] P. Compton, P. Kehoe, K. Sinha, M. A. Torrington, and W. Ling, "Gabapentin improves cold-pressor pain responses in methadone-maintained patients," Drug and Alcohol Dependence, vol. 109, no. 1-3, pp. 213-219, 2010.

[60] J. W. Norton, "Gabapentin withdrawal syndrome," Clinical Neuropharmacology, vol. 24, no. 4, pp. 245-246, 2001.

[61] F. Barrueto Jr., J. Green, M. A. Howland, R. S. Hoffman, and L. S. Nelson, "Gabapentin withdrawal presenting as status epilepticus," Journal of Toxicology, vol. 40, no. 7, pp. 925-928, 2002.

[62] K. T. Tran, D. Hranicky, T. Lark, and N. J. Jacob, "Gabapentin withdrawal syndrome in the presence of a taper," Bipolar Disorders, vol. 7, no. 3, pp. 302-304, 2005.

[63] M. Rowbotham, N. Harden, B. Stacey, P. Bernstein, and L. Magnus-Miller, "Gabapentin for the treatment of postherpetic neuralgia: a randomized controlled trial," Journal of the American Medical Association, vol. 280, no. 21, pp. 1837-1842, 1998.

[64] J. Mao and L. L. Chen, "Gabapentin in pain management," Anesthesia and Analgesia, vol. 91, no. 3, pp. 680-687, 2000. 


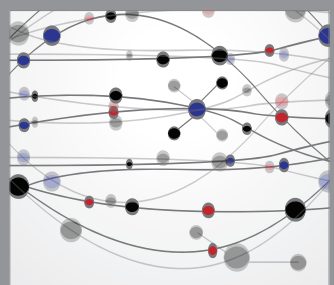

The Scientific World Journal
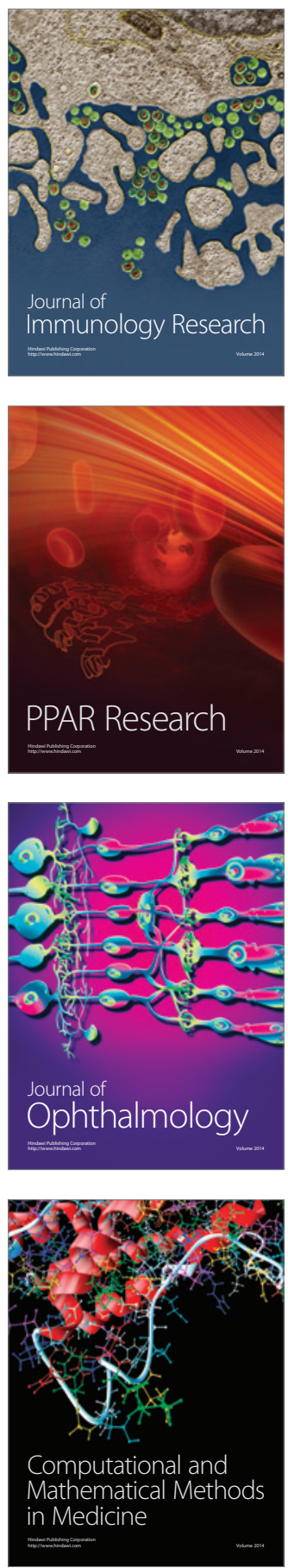

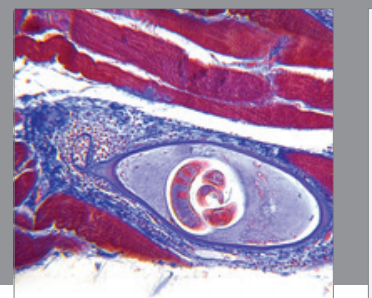

Gastroenterology

Research and Practice
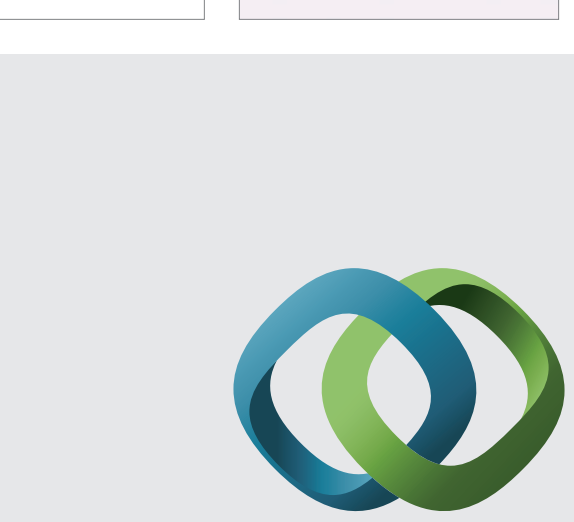

\section{Hindawi}

Submit your manuscripts at

http://www.hindawi.com
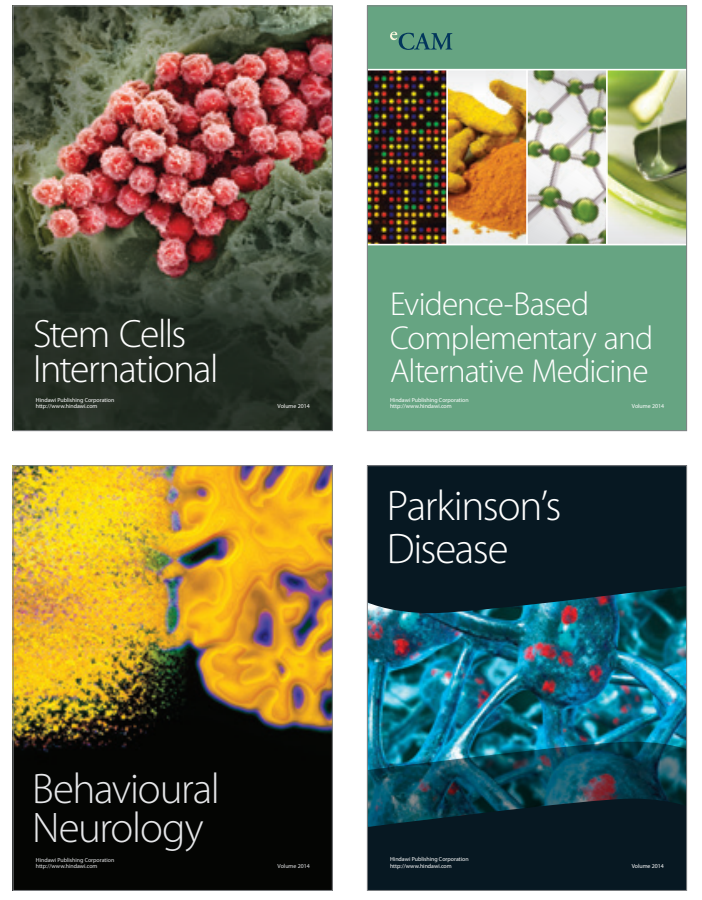
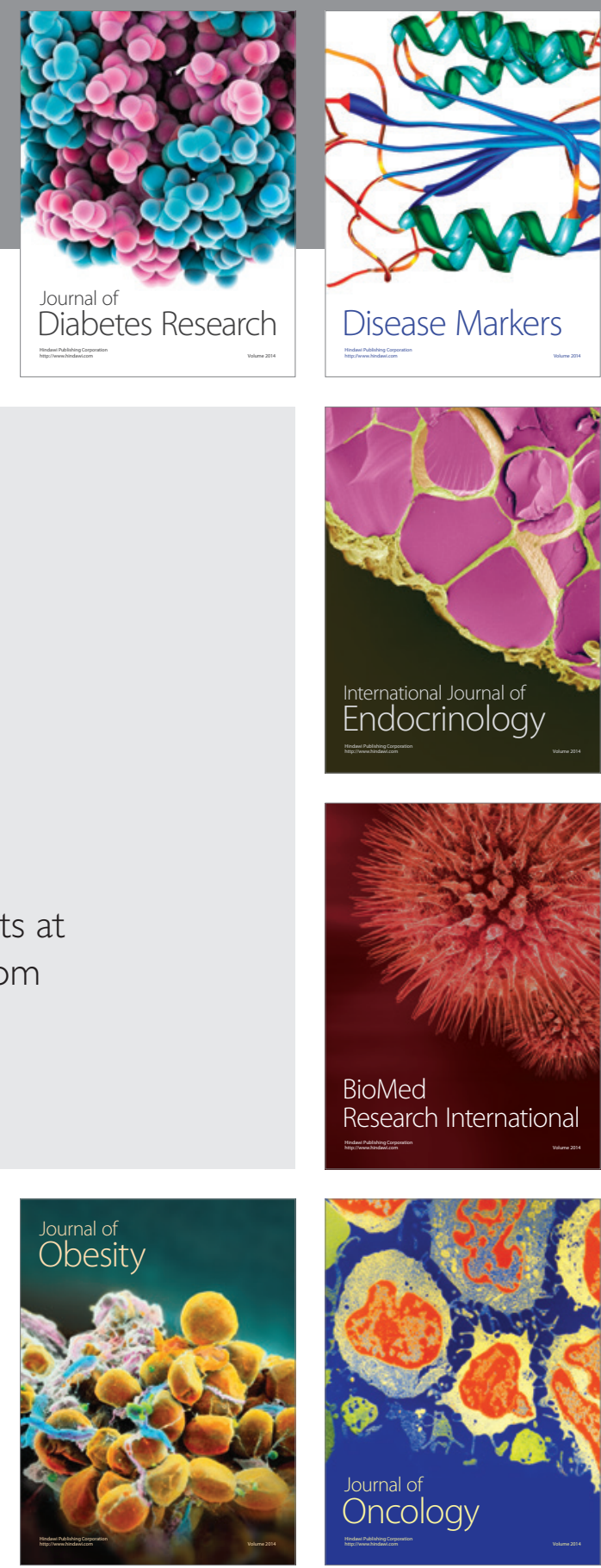

Disease Markers
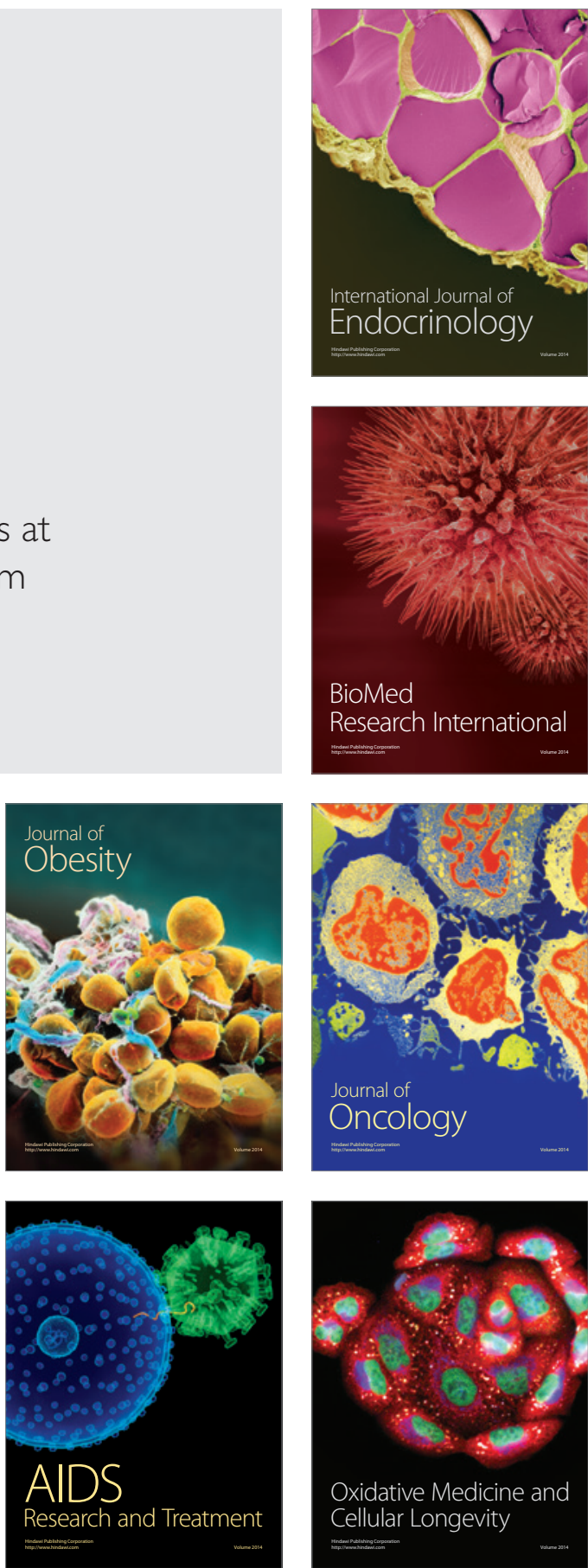